\title{
Increased mitochondrial distribution in early-cleaving embryos indicate successful pre-implantation development
}

\author{
Nor Shahida Abdul Rahman a, b, Mimi Sophia Sarbandi a, Wan Hafizah Wan Jusof a, \\ Zolkapli Eshak a, c, Salina Othman a, d, Fathiah Abdullah a, Yuhaniza Shafinie Kamsani a,

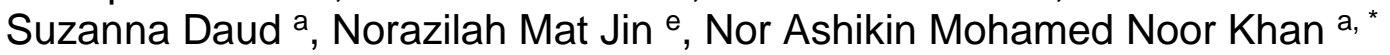 \\ a Maternofetal and Embryo Research Group (MatE), Faculty of Medicine, Universiti Teknologi MARA, Selangor Branch, Sungai Buloh Campus, \\ 47000 Sungai Buloh, Selangor, Malaysia \\ b Faculty of Medicine and Health Sciences, Universiti Putra Malaysia, 43400 UPM Serdang, Selangor, Malaysia \\ c Faculty of Pharmacy, Universiti Teknologi MARA, Selangor Branch, Puncak Alam Campus, 42300 Bandar Puncak Alam, Selangor, Malaysia \\ d Institute of Medical Molecular Biotechnology (IMMB), Faculty of Medicine, Universiti Teknologi MARA, Selangor Branch, Sungai Buloh Campus, \\ 47000 Sungai Buloh, Selangor, Malaysia \\ ${ }^{e}$ Faculty of Medicine, Universiti Teknologi MARA, Selangor Branch, Sungai Buloh Campus, 47000 Sungai Buloh, Selangor, Malaysia
}

* Corresponding author: noras011@salam.uitm.edu.my

\section{Article history}

Received 20 March 2018

Revised 5 April 2018

Accepted 8 May 2018

Published Online 16 December 2018

\section{Graphical abstract}

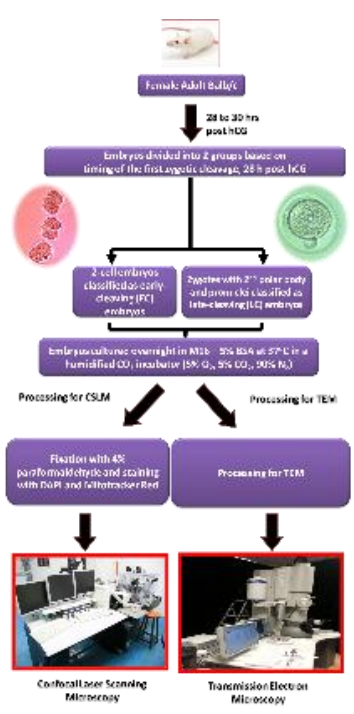

\section{Abstract}

The timing of the first zygotic cleavage is an accurate predictor of embryo quality. Embryos that cleaved early have higher developmental viability compared to their late counterparts. During embryonic development, cleavage is affected by cellular metabolic processes performed by mitochondria and its synergistic interaction with the endoplasmic reticulum (ER). However, in depth study on differences of mitochondria and ER ultrastructures in early- cleaving (EC) versus latecleaving (LC) embryos is limited. This study compares mitochondria and ER ultrastructures of EC versus LC embryos using Confocal Laser Scanning Microscopy (CLSM) and Transmission Electron Microscopy (TEM). Embryos were obtained from female ICR superovulated mice, 28-30 hours post hCG. Two-cell embryos were categorized as early-cleaving (EC), while zygotes with the second polar body and two pronuclei present were categorized as late-cleaving (LC). The LC embryos were cultured in vitro until the 2- cell stage. In EC embryos, mitochondria were mostly found at the perinuclear region and closely associated with dense ER. Meanwhile, mitochondria of LC embryos were distributed uniformly within the cytoplasm. Mitochondrial fluorescence intensity was significantly higher in EC versus LC [(18.7 \pm 0.4$)$ versus $(14.6 \pm 0.4)] \times 10^{5}$ pixel, $(p<0.01)$. Development to the blastocyst stage was also significantly higher in EC compared to LC embryos $(96.7 \%$ versus $60.9 \%)$ $(p<0.01)$. Higher viability of EC embryos is attributed to the close association of their mitochondria to ER. This contributed to better mitochondrial fission, resulting in enhanced energy generating processes and preimplantation development.

Keywords: Embryonic development, mitochondria, endoplasmic reticulum, confocal laser scanning microscopy, transmission electron microscopy

\section{INTRODUCTION}

Most Assisted Reproductive Technology (ART) establishments are moving towards single embryo transfer as it excludes the complications to multiple pregnancies. The search for reliable noninvasive techniques in assessing embryo quality has become increasingly important to achieve successful implantation of a single good quality embryo. The timing of the first zygotic cleavage is reported as an alternative indicator of embryo quality (Francsovits et al., 2005; Fu et al., 2009; Nielsen and Ali 2010). It has been associated with parameters that may affect embryonic development (Van Soom A et al., 1997). Studies have shown that embryos which cleaved earlier during the first zygotic cleavage had higher developmental viability, higher rates of blastocyst formation, pregnancy and live birth (Lundin et al., 2001; Lechniak et al., 2008; Nielsen and Ali 2010; Isom et al.
2012). Hiragii and Solter (2004) in their study reported that the entry point of sperm controls the first cleavage of the mouse zygote. Although embryo selection based on the timing of first zygotic cleavage has become a common practice in IVF laboratories, factors that contribute to the superiority of early cleavers have yet to be established.

Mitochondria are important cellular organelles involved in ATP synthesis and metabolic assimilation, reactive oxygen species (ROS) production and maintenance as well as apoptosis regulation. With regards to reproduction, they play many crucial roles in gametogenesis and fertilization as well as cellular development in terms of energy production via oxidative phosphorylation. Mitochondria are able to initiate apoptotic cell death by regulating caspase activation through outer membrane permeabilization (Tait and Green, 2013). The control of apoptosis from the mitochondria ensures production of viable gametes and ROS signalling (Ramalho-Santos and Amaral, 2013). As 
such, they are organelles of major importance in the oocyte and early embryo that interact synergistically with the endoplasmic reticulum (ER) in the regulation of mitochondrial fission (de Brito and Scrorrano, 2008), calcium transfer (Csordas et al., 2010), autophagy (Axe et al., 2008; Hamasaki et al., 2013) and inflammation (Horner et al., 2011). Perturbations in their association could negatively affect embryo quality. In this study, mitochondria were a major focus because changes in the distribution of mitochondria that supply the majority of cellular ATP are very critical for the maturation and development of the oocytes and embryos (Yu et al. 2010). When embryos are unable to produce an adequate amount of energy, anomalies can occur which lead to changes in the activity and the structural organization of the cell and mitochondria, in particular. Such instabilities can lead to profound diseases and metabolic disorders. With regards to the crucial role of mitochondria in pre-implantation development, this study was conducted to compare mitochondrial distribution and intensity of early cleaving embryos versus late cleaving embryos using CLSM and TEM.

\section{EXPERIMENTAL}

Female ICR mice, aged $6-8$ weeks old were induced to superovulate by intraperitoneal injection of 5 IU of Pregnant Mare Serum Gonadotrophin (PMSG; Folligon, Intervet) followed by 5 IU Human Chorionic Gonadotrophin (HCG; Chorulon, Intervet) $48 \mathrm{~h}$ later. The females were mated with fertile males and those showing the presence of vaginal plug were euthanized after 28-30 h of HCG administration. Embryos were flushed out of oviducts using M2 medium (Sigma, USA). The embryos were graded based on Khalili and Anvari (2007). Embryos with 2-cells at 28-30 hours post hCG were categorized as EC while zygotes with the presence of the second polar body and two pronuclei were categorized as LC embryos. The embryos were cultured in M16 culture medium (Sigma, USA) + 3\% Bovine Serum Albumin (BSA; Sigma, USA) in a $\mathrm{CO}_{2}$ incubator $\left(37^{\circ} \mathrm{C}, 5 \%\right.$ $\mathrm{CO}_{2}$ ). Both EC and LC embryos were processed for CLSM and TEM. All animal procedures were approved by the Institutional Animal Care and Use Committee (Approval code ACUC-7/11).

The embryos were fixed in $4 \%$ formaldehyde and incubated for 24 h. Permeabilization was then done with $1 \%$ Triton-X in Phosphate Buffer Saline (PBS) followed by labelling with MitoTracker Red (Molecular Probes, USA). They were washed twice with PBS, mounted on slides with ProLong Gold antifading agent (Invitrogen, USA), and stored overnight at $4^{\circ} \mathrm{C}$. The slides were observed using CLSM [Leica TCS SP5 AOBS, Germany] with excitation at $543 \mathrm{~nm}$ and emission at $560 \mathrm{~nm}$. Quantitative analyses of fluorescent images were performed using LAS AF Lite software.

For ultrastructural assessment, the 2 -cell embryos were fixed in $2.5 \%$ glutaraldehyde at $4^{\circ} \mathrm{C}$ and rinsed with cacodylate buffer for 10 minutes each for three changes at room temperature before fixation in $1 \%$ osmium oxide for 2 hours at $4^{\circ} \mathrm{C}$. This rinsing procedure was repeated. The serial dehydration in acetone was performed before they were placed in beam capsules. Agar 100 resin-acetone mixtures were added into the capsules overnight. Finally, the mixture was replaced by $100 \%$ resin before being oven-dried at $60^{\circ} \mathrm{C}$ for 48 hours. Each sample was sectioned and stained with uranyl acetate and lead citrate, before being viewed under the Transmission Electron Microscope (TEM) (Tecnai G2).

Statistical analysis was performed using the SPSS version 22. The development of embryos to the blastocyst stage was documented as percentages. The difference between the two groups (EC and LC embryos) was analyzed using Chi-square test. Assessment of mitochondrial distributions was based on the intensity of fluorescent probes after staining. Independent T-test was performed to analyze the difference of intensities among EC and LC embryos. Data were plotted as mean $\pm \mathrm{SD}$. The $\mathrm{P}$ value of less than 0.01 was considered statistically significant. Meanwhile, descriptive analysis was carried out to assess changes in the ultrastructure of the EC and LC embryos.

\section{RESULTS AND DISCUSSION}

There were significant differences between EC and LC embryos at 4 - cell $(98.3 \%$ vs $71.8 \%)$, morula $(98.3 \%$ vs $67.8 \%)$ and blastocyst
$(96.7 \%$ vs $60.9 \%)$ stages $(\mathrm{p}<0.01)$ (Table1). Previously, EC embryos have been reported to have higher developmental competence and quality than LC embryos (Kaith et al., 2015). Our study supports this finding. Our data demonstrated a reduction of fluorescent intensity produced by the LC embryos which also indicated the possibility of less number of active mitochondria as demonstrated by Dumollard et al., 2009. A reduction in active mitochondrial function has been described to decrease ATP output in the pre-compacting embryo which inevitably influences the offspring phenotype (Zander et al., 2015).

Table 1 Preimplantation development of EC and LC embryos.

\begin{tabular}{|c|c|c|c|c|}
\hline \multirow{2}{*}{$\begin{array}{l}\text { Embryo } \\
\text { Groups }\end{array}$} & \multicolumn{4}{|c|}{ No. of embryos (\%) } \\
\hline & $\begin{array}{l}\text { 2-cell } \\
\text { stage } \\
(24 \mathrm{~h})\end{array}$ & $\begin{array}{c}\text { 4-cell stage } \\
(48 \mathrm{~h})\end{array}$ & $\begin{array}{c}\text { morula } \\
\text { stage }(72 \mathrm{~h})\end{array}$ & $\begin{array}{l}\text { blastocyst } \\
\text { stage }(96 \mathrm{~h})\end{array}$ \\
\hline EC & $60(100)$ & $59(98.3)^{*}$ & $59(98.3)^{*}$ & $58(96.7)^{\star}$ \\
\hline LC & $174(100)$ & $125(71.8)$ & $118(67.8)$ & $106(60.9)$ \\
\hline
\end{tabular}

The intensity of Mito Tracker Red fluorescence is shown in Fig. 1. Fluorescence intensity of mitochondria in EC embryos was significantly higher compared to LC embryos $(18.7 \pm 0.4$ vs $14.6 \pm 0.4)$ x $10^{5}$ pixels $(\mathrm{p}<0.01)$.

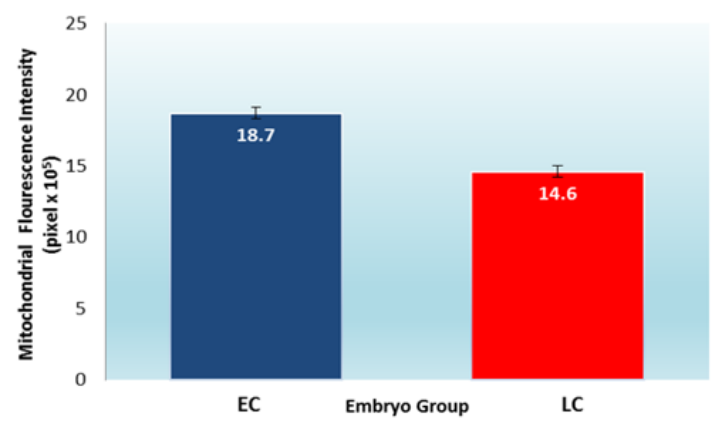

Fig. 1 Mitochondria fluorescence intensities in (EC) vs (LC) embryos at 2-cell stage (Mean $\pm S D)$. $(p<0.01)$.

The mitochondria of EC embryos were more intense and distinct when compared with LC embryos (Fig. 2A and Fig. 2B). They were distributed at the periphery of the nucleus and localised towards the centre of the blastomere (Fig. 2A). On the other hand, LC embryos were characterized by the uniform distribution of mitochondria throughout the cytoplasm (Fig. 2B) in agreement with earlier findings (Wilding et al., 2001).

The uniform distribution of mitochondria observed in LC embryos suggested that alleviation of metabolism has occurred. Both EC and LC embryos had mitochondria with the round shape of moderate swelling and few cristae, consistent with early embryos.
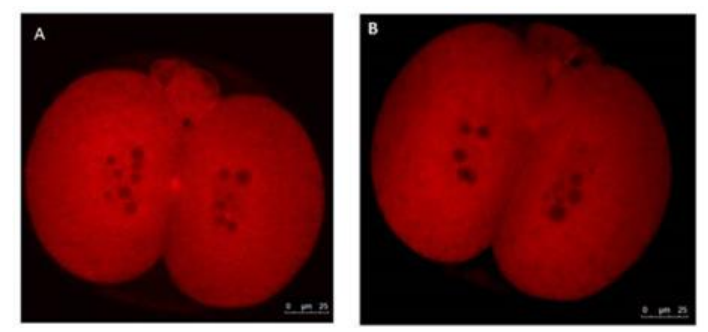

Fig. 2 MitoTracker Red fluorescence intensities in EC embryos (A) compared to LC embryos (B).

The ultrastructural findings of EC and LC embryos are shown in Fig. 3 and Fig. 4. At 2- cell stage, mitochondria had round to ovoid forms with few cristae, localized around the nucleus (Fig. 3A). 
Meanwhile, fewer mitochondria were uniformly distributed in LC embryos (Fig. 4C). Presence of vacuolated mitochondria was observed in both EC and LC embryos. However, such forms were less distinct in EC embryos compared to LC embryos. In EC embryos (Fig. 3B), endoplasmic reticulum (ER) were found more densely distributed in the cytoplasm, alongside the mitochondria compared to LC embryos (Fig. 4D). An earlier study has described active mitochondrial migration which surrounds pronuclei to form a pattern that persists through early cleavage stages, such as the 2-cell stage (Dumollard et al., 2009). It is an important part of normal development. Treatments to embryos may affect the normal pattern of mitochondrial distribution and impair development. Our results suggest that the decreasing number of both EC and LC embryos in culture over time may be due to decreased mitochondrial function based on its distribution pattern in the cell. The localization and segregation of mitochondria to blastomeres in the cleaving embryo are strictly regulated. In the CSLM images, the high mitochondrial intensity of EC embryos indicates active mitochondria localized at the periphery of the nucleus. This is in agreement with the observed TEM ultrastructures of mitochondria. Perinuclear clustering of mitochondria supports cell division and preimplantation development. This is in agreement with other studies (Van Blerkom, 2011; Nagai et al., 2006; Suzuki et al., 2006; Wilding, 2001).
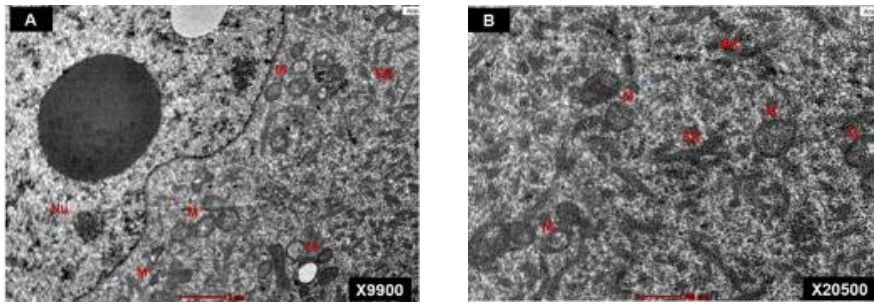

Fig. 3 Mitochondria (M), Endoplasmic Reticulum (ER) and Nucleus (Nu) of EC (Fig. 3A) and LC (Fig. 3B) embryos.
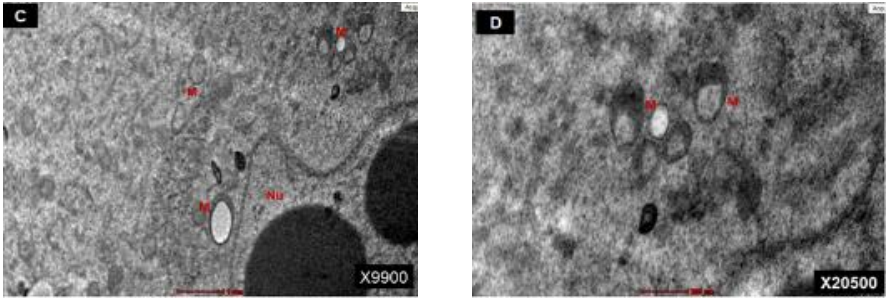

Fig. 4 Mitochondria (M), Endoplasmic Reticulum (ER) and Nucleus (Nu) of EC (Fig. 4C) and LC (Fig. 4D) embryos.

\section{CONCLUSION}

The timing of zygotic cleavage was found to be related to mitochondrial distribution and density during early preimplantation stages. Higher mitochondrial density and localised distribution in EC embryos contributed to better mitochondrial fission, resulting in enhanced energy generating processes and preimplantation development.

\section{ACKNOWLEDGEMENT}

The authors gratefully acknowledge the Research Management Institute (RMI), Universiti Teknologi MARA, UiTM of Malaysia in providing the BESTARI Grant (0014/2016) and Malaysian Ministry of Higher Education in providing the RAGS grant (46/2015) We also thank the staff of the Imaging Unit for the facility provided from Faculty of Pharmacy Universiti Teknologi MARA, UiTM Puncak Alam Campus, Malaysia.

\section{REFERENCES}

Axe, E.L., Walker, S.A., Manifava, M., Chandra, P., Roderick, H.L., Habermann, A., Griffiths, G., Ktiskatis, N. T. (2008). Autophagosome formation from membrane compartments enriched in phosphatidylinositol 3-phosphate and dynamically connected to the endoplasmic reticulum. Journal of Cell Biology 182: 685-701.

Csordas, G., Varnai, P., Golenar, T., Roy, S., Purkins, G., Schneider, T.G., Balla, T., Hajnoczky, G. (2010). Imaging interorganelle contacts and local calcium dynamics at the ER-mitochondrial interface. Molecular Cell 39: 121-132.

De Brito O.M., and Scorrano L. (2008). Mitofusion 2 tethers endoplasmic reticulum to mitochondria. Nature 456: 605-610.

Dumollard, R., Carroll, J., Duchen, M. R., Campbell, K., and Swann, K. (2009). Mitochondrial function and redox state in mammalian embryos. Seminars in Cell \& Development Biology 20: 346-353.

Francsovits, P., Toth, L., Takacs, F., Murber, A., Papp, Z. and Urbancsek, J. (2005). Early pronuclear breakdown is a good indicator of embryo quality and viability. Fertility and Sterility 84(4): 881-887.

Fu, J., Wang, X.J., Wang, Y.W., Gemzell-Danielsson, K. and Sun, X.X. (2009). The influence of early cleavage on embryo developmental potential and IVF/ICSI outcome. Journal Assisted Reproduction Genetics 26: 437-441.

Hamasaki, M., Furuta, N., Matsuda, A., Nezu, A., Yamamoto, A., Fujita, N., Oomori, H., Noda, T., Haraguchi, T., Hiraoka, Y., Amano, A., Yoshimori, T. (2013). Autophagosomes form at ER-mitochondria contact sites. Nature 495: 389-393.

Hiiragi, T. and Solter, D. (2004). First cleavage plane of the mouse egg is not predetermined but defined by the topology of the two apposing pronuclei. Nature 430: 360-364.

Horner, S.M., Liu, H.M., Park, H.S., Briley, J., Gale Jr. M. (2011). Mitochondrial-associated endoplasmic reticulum membranes (MAM) from innate immune synapses and are targeted by hepatitis $\mathrm{C}$ virus. Proceedings of the National Academy of Sciences 108: 14590-14595.

Isom, S. C., Li, R. F., Whitworth, K. M. and Prather, R. S. (2012). Timing of first embryonic cleavage is a positive indicator of the in vitro developmental potential of porcine embryos derived from in vitro fertilization, somatic cell nuclear transfer and parthenogenesis. Molecular Reproduction and Development 79(3): 197-207.

Kaith, S., Saini, M., Raja, A.K., Sahare, A.A., Jyotsana, B., Madheshiya, P., Palta, P., Chauhan, M.S., Manik, R.S. and Singla, S.K. (2015). Early cleavage of handmade cloned buffalo (Bubalus bubalis) embryos is an indicator of their developmental competence and quality. Reproduction Domestic Animals 50(2): 214-20. doi: 10.1111/rda.12472.

Lechniak, D., Pers-Kamcyc, E. and Pawlak, P. (2008). Timing of first zygotic cleavage as a marker of developmental potential of mammalian embryo. Reproduction Biology 8(1): 23-42.

Lundin, K., Bergh, C. and Hardarson, T. (2001). Early embryo cleavage is a strong indicator of embryo quality in human IVF. Human Reproduction 16: 2652-2657.

Khalili, M.A., and Anvari., M. (2007). The effect of in vitro culture on cleavage rates and morphology of in-vivo developed embryos in mice. Iranian Journal of Reproductive Medicine 1: 17-22

Nagai, S., Mabuchi, T., Hirata, S., Shoda, T., Kasai, T., Yokota, S., Shitara, H., Yonekawa, H. and Hoshi, K. (2006).Correlation of abnormal mitochondrial distribution in mouse oocytes with reduced developmental competence.Tohoku Journal of Experimental Medicine 210(2): 137-144.

Nielsen, H.I. and Ali, J. (2010). Embryo culture media, culture techniques and embryo selection: A tribute to Wesley Kingston Whitten. Journal Reproduction Stem Cell Biotechnology (1): 1-29.

Ramalho-Santos, J. and Amaral, S. (2013). Mitochondria and mammalian reproduction. Molecular And Cellular Endocrinology 379(1-2): 74-84.

Suzuki, H., Satoh, M. and Kabashima, K. (2006) Distributions of mitochondria and the cytoskeleton in hamster embryos developed in vivo and in vitro. Journal of Mammalian Ova Research 23: 128-134.

Van Blerkom, J. (2011). Review: Mitochondrial function in human oocyte and embryos and their role in developmental competence. Mitochondrion 11: 797-813.

Van Soom A., Marie-Therese Y., and De Kruif A. (1997). Relationship between timing of development, morula morphology and cell allocation to inner cell mass and trophectoderm in vitro-produced bovine embryos. Molecular Reproduction and Development 47: 47-56

Wilding, M., Dale, B., Marino, M., di Matteo, L., Alviggi, C., Pisaturo, M.L., Lombardi, L. and De Placido, G. (2001). Mitochondrial aggregation patterns and activity in human oocytes and preimplantation embryos. Human Reproduction 16(5): 909-917.

Yu Y., Remi D., Andreas R., Lai F.A., and Karl S. (2010). Redistribution of mitochondria leads to burst of ATP production during spontaneous mouse oocyte maturation. Journal of Cellular Physiology 224(3): 672-680.

Zander-Fox, D.L., Fullston, T., McPherson, N.O., Sandeman, L., Kang, W.X., Good, S.B., Spillane, M. and Lane, M. (2015). Reduction of Mitochondrial Function by FCCP During Mouse Cleavage Stage Embryo Culture Reduces Birth Weight and Impairs the Metabolic Health of Offspring. Biology of Reproduction 92(5): 124 